\title{
Préservation de la morphologie, chez les malades Alzheimer au stade sévère de la maladie
}

\author{
Devevey Alain \\ Université de Franche Comté \\ Pôle Contextes, Langages, Didactiques \\ ELLIADD EA 4661 \\ UFR SMP Orthophonie
}

Résumé. L'objet de cette communication sera de montrer comment une compétence morphologique implicite résiste à l'avancée de la maladie d'Alzheimer. Nous illustrerons notre propos par les résultats de deux études visant à étudier les capacités sémantiques des patients, à travers la compréhension et la production des marques morphologiques. Nous chercherons à aborder la catégorisation non pas d'après un modèle taxinomique (Rosch), mais à travers les informations véhiculées par la morphologie. Nous nous appuierons sur des études montrant que les propriétés des signes linguistiques notamment, déterminent la structuration des catégories (Dubois et Poitou). Nous montrerons que les patients atteints de maladie d'Alheimer utilisent l'information morphologique (lexicale et dérivationnelle) pour réaliser une tâche d'inclusion catégorielle. A cette fin nous avons proposé à 30 patients MA ainsi qu'à 30 témoins, une tâche d'inclusion catégorielle. Nous avons créé un protocole expérimental constitué d'items faisant intervenir 3 variables : (1) appartenance ou non à la catégorie "Arbre" ou "Métier", (2) avec ou sans morphème dérivationnel "-ier" et (3) morphème lexical lié (ou pseudo-lié) ou non lié au fruit/fonction. L'objectif était de mettre en exergue les effets respectifs du morphème lexical et du morphème dérivationnel. L'analyse des résultats révèle que l'information conjuguée portée par morphème lexical et le morphème dérivationnel "-ier" est opérante, pour les malades comme pour les témoins. Les malades utilisent davantage les informations portées par la langue que les témoins. Il existe donc une robustesse de la morphologique, en mémoire implicite, qui permet aux sujets de répondre à la tâche d'inclusion catégorielle. Ainsi, les difficultés lexico-sémantiques des patients ne se posent pas uniquement en terme de difficulté d'accès ou de perte des représentations sémantiques ainsi que le suppose la psychologie cognitive, puisque patients et témoins peuvent traiter les items comme signes linguistiques. La deuxième étude que nous présenterons est centrée sur la production des néoformes, à partir de l'analyse de corpus d'une patiente porteuse de la Maladie d'Alzheimer au stade sévère. Nous tenterons de montrer comment ces manifestations, dont le nombre augmente avec l'avancée de la maladie sont à considérer, non pas comme la marque d'une désintégration irréversible du langage mais comme de véritables mécanismes de compensation, qui revêtent les caractéristiques morpholexicales et morphosyntaxiques du code linguistique. Ces deux études tendent à démontrer que les productions, apparemment dépourvues de sens, peuvent en réalité être considérées comme des idiolectes. Ces idiolectes restent dépendants, même au stade ultime de l'évolution de la maladie, des règles du code linguistique, implicitement construites par chaque sujet. Les troubles du langage, dans le cadre des maladies neurodégénératives, ne peuvent donc être considérés sous l'aspect d'une "perte du langage" voire d'un "désapprentissage de la langue".

\begin{abstract}
Conservation of the morphology, by people with Alzheimer' disease, at the severe stage. The aim of this communication is to show how Alzheimer's disease does not disrupt the implicit morphological knowledge. We present two studies in order to reveal the semantic abilities of patients, through both understanding and producing morphological markers. We consider categorization process, not from a taxonomic model (Rosch), but through the information conveyed by morphology. According to this model, the properties of linguistic signs determine the structure of categories (Dubois and Poitou). We will try to show that people with Alheimer's Disease (AD) use morphological information (lexical and derivational) to succeed in doing a categorical inclusion task. We proposed to $30 \mathrm{AD}$ people and 30 matched healthy subjects, a task of category-specific inclusion. We created an experimental protocol constituted by items bringing in 3 variables: (1) membership or not in the category "Tree" or "Work", (2) with or without derivational morpheme-" ier " and (3)
\end{abstract}




\begin{abstract}
lexical bound (or pseudo-bound) morpheme or not bound to the fruit / function. The aim was to highlight the respective effects of the lexical morpheme and the derivational morpheme. The results show that the combined information carried by lexical morpheme and the derivational morpheme-" ier " is effective, for the AD people as for the matched healthy subjects. People with AD use more the information carried by the language than the others. The results point out a resilience of morphology, in memory implicit, which allows the subjects to succeed the task of category-specific inclusion. So, the lexico-semantic difficulties of the patients do not depend only on difficulty of access or loss of the semantic representations as well as supposes it cognitive psychology, because AD patients can process items as linguistic signs, a well as do healthy subjects. The goal of second research is to study production of neoforms, through the analysis of corpus of an AD patient at the severe stage. We shall try to show how the production of these terms increases with the advance of the disease. Furthermore, these productions are to be considered not as mark of an irreversible destruction of the language but as of real mechanisms of compensation, which take on the morpholexicales and morphosyntactic characteristics of the linguistic code. These results tend to demonstrate that the productions, apparently meaningless, can be considered in reality as idiolects. This idiolects respect the rules of the linguistic code, implicitly built by each one, even at the ultimate stage of the disease. Thus, speech difficulties, cannot be considered as a " loss of the language " and nor a "regress of language".
\end{abstract}

\title{
1 Introduction
}

Concernant les troubles du langage dans la maladie d'Alzheimer, le paradigme dominant en psycholinguistique comme en psychologie cognitive, se fonde sur la théorie innéiste du langage (Chomsky, 1955, 2011). Partant, une écrasante majorité d'études dans ces deux domaines cherche à étudier la détérioration d'un langage considéré comme une "fonction cérébrale". La manifestation essentielle des troubles langagiers étant le manque du mot, ces études portent majoritairement sur le - ou les - dysfonctionnement(s) cérébral(aux) à l'origine des troubles lexicaux. On trouve néanmoins dans la littérature des exemples mentionnant des "capacités syntaxiques" préservées chez les personnes atteintes de la maladie (Berrewaerts, Hupet, \& Feyereisen, 2002; Kavé \& Lévy, 2004; Kempler, Curtiss, \& C., 1987; Murdoch \& Chenery, 1987). Ainsi, la partition entre lexique et syntaxe inspirée de la théorie modulaire du langage (Fodor, 1983) s'impose comme une évidence. Néanmoins, le rôle et l'évolution des compétences morphologiques au cours de la maladie reste largement négligé - voir pour synthèse à ce sujet (M. Barkat-Defradas \& Gayraud, 2013; M Barkat-Defradas, Martin S., Rico-Duarte, \& Brouillet, 2008). Par ailleurs, beaucoup plus rares sont les travaux qui tentent de sortir du paradoxe selon lequel la "fonction langage" serait atteinte dans certains modules et pas dans d'autres (A Devevey, 2008; A. Devevey, 2009a).

\section{Le traitement cognitif du langage}

Nous tenterons une approche, à nos yeux plus prometteuse, qui envisage le langage non pas comme un instrument ni une fonction cognitive, mais comme le milieu au sein duquel nous évoluons (Rastier, 2003) De ce point de vue, dans la lignée des travaux de Nespoulous (Nespoulous, 1994, 2004) nous considérons que la seule conséquence directe de l'atteinte du substrat neuroanatomique (les lésions neurologiques) est une atteinte des fonctions cognitives ${ }^{1}$. Cette atteinte va perturber chez le locuteur le traitement du "milieu langage", tout comme elle perturbera le traitement cognitif des autres milieux dans lesquels il évolue. Pour ce qu'il en est de la spécificité du traitement du langage, nous nous plaçons dans la perspective d'un modèle duel, qui suppose non pas une partition modulaire du langage, mais plutôt une sollicitation différentielle des fonctions cognitives, et sur le plan mnésique, de la mémoire déclarative ou de mémoire procédurale en particulier, selon le type de traitement cognitif spécifique qu'imposent les usages particuliers de ce milieu (Macoir \& Fossard, 2008; Paradis, 1994, 2004). Nous élargissons, pour notre part, le propos sur le plan sémantique à un traitement en mémoire déclarative de la sémantique lexicale 
référentielle vs un traitement en mémoire procédurale de la sémantique interprétative au sens de Rastier (Rastier, 1987). Les processus interprétatifs étant mémorisés à partir de la fréquence et de la variété des occurrences interprétatives rencontrées par le locuteur dans le milieu des usages auxquels il est confronté.

Notre postulat est le suivant : si le langage n'est pas un instrument ni une fonction, alors deux évidences s'imposent. D'une part les dysfonctionnements observés ont une autre cause que l'altération de la "fonction langage" et d'autre part en modifiant les protocoles d'observation, voire en modifiant les méthodes d'analyses des résultats obtenus par les protocoles classiques, on doit pouvoir mettre en lumière des évidences de fonctionnements langagiers non canoniques mais issus de traitements cognitifs fonctionnels du "milieu langage".

A cet égard la maîtrise de la morphologie s'avère un domaine d'étude particulièrement prometteur. En effet, celle-ci n'apparaît pas comme dépendante d'un apprentissage explicite de la part du sujet (Paradis, op. cit.). Au contraire, le locuteur va élaborer implicitement des stratégies d'interprétation de cooccurrences de morphèmes lexicaux et grammaticaux du type "nom de fruit + ier" = "arbre portant des fruits". Ce parti-pris théorique nous permet d'éviter d'entrer dans le débat : modèles duels vs. modèles à voie unique (Baayen, 2007; S. Pinker, 1999; S. Pinker \& Ullman, 2002), essentiellement argumenté à partir des aspects réguliers vs irréguliers de la morphologie verbale flexionnelle. Le paradigme "régulier" vs "irrégulier" suppose l'existence d'une régularité objective. Or dans ce domaine comme dans celui des usages langagiers, la variation est la règle. En effet, si "cerisier" est bien un "arbre à cerises", "peuplier" n'en est pas pour autant "un arbre à peuple" (Poitou \& Dubois, 1999). Pourtant nul doute que n'importe quel locuteur assimile cette contradiction apparente et interprète correctement "peuplier" en contexte. Si ces phénomènes ont été décrits sur le plan linguistique, ils ne l'ont pas été autant sur le plan cognitif. Néanmoins si on suppose une "régularité implicite", construite par le locuteur à partir des occurrences que lui offre le milieu langagier auquel il est confronté, alors on doit pouvoir mettre en évidence une différence de statut entre "cerisier" et "peuplier". Ce dernier étant cognitivement plus "coûteux", du fait de la transgression de la régularité "nom de fruit + ier", imposée par l'association "peupl + ier". On peut supposer, comme le suggère Rastier, que l'influence du contexte vienne lever cette apparente ambigüité chez un locuteur ordinaire, mais peut-être est-il possible d'observer des traces expérimentales de ce phénomène. C'est ce qui constituera l'objet de notre première étude.

\section{Morphologie et catégorisation}

A ce titre, revisiter les tâches de classification catégorielle revêt un intérêt à la fois sémantique et morphologique. Classiquement, celle-ci est considérée par les cognitivistes et les psycholinguistes à la suite de (Rosch, Mervis, Gray, Johnson, \& Boyes-Braem, 1976) comme un état de fait naturel ${ }^{2}$ reflété par le "sens des mots" et que les locuteurs intègrent à partir d'indices perceptifs. C'est ce paradigme qui demeure le support de toutes les études portant sur la détérioration du langage dans le cadre des maladies neurodégénératives.

Pour notre part, à la suite des travaux de D. Dubois (D. Dubois, 1991), nous envisageons la catégorisation comme un phénomène éminemment subjectif, dont les principes de construction dépassent de beaucoup une simple élaboration à partir d'indices perceptifs (Rossi, 2013). En français, comme dans d'autres langues en effet, la morphologie apparaît également comme un des fondements des processus de classification (D. Dubois \& Poitou, 2002; Grinevald-Graig, 1986; Poitou \& Dubois, 1999). Ces processus sont implicitement élaborés et mémorisés par les locuteurs. La maladie d'Alzheimer altérant les processus mnésiques explicites tout en épargnant la mémoire implicite, ces processus implicites vont résister à l'avancée de la détérioration neuroanatomique. Autrement dit, proposer une tache de catégorisation selon le paradigme classique (inclusion catégorielle, fluence catégorielle) conduira inévitablement les sujets atteints de maladie d'Alzheimer à l'échec, alors que leur soumettre une tâche de même nature, mais qui sollicitera leur potentiel implicite de traitement du langage leur offrira davantage de chances de réussite. Nous pourrons ainsi mettre en évidence la robustesse des capacités de traitement de la morphologie chez les sujets atteints de la maladie d'Alzheimer. 


\section{Expérience 1 : Préservation de la morphologie chez les sujets atteints de maladie d'Alzheimer}

\subsection{Description de l'échantillon}

Les critères d'inclusion étaient les suivants : diagnostic de maladie d'Alzheimer confirmé, être de langue maternelle française, avoir une perception auditive suffisante, ne pas souffrir d'agnosie auditive, ne pas avoir d'antécédents alcooliques, ne pas avoir été victime d'accident vasculaire cérébral, ne pas bénéficier de traitement à base de neuroleptique. La population cible, se composait de 15 personnes atteintes d'une maladie d'Alzheimer : douze femmes âgées de 69 ans à 90 ans et trois hommes âgés de 85 à 86 ans. Leurs scores au MMSE (Folstein, Folstein, \& P.R., 1975).

Les 15 sujets témoins appariés ont été sélectionnés à partir des mêmes critères d'inclusion que les personnes atteintes de maladie d'Alzheimer. Toutefois, pour ces derniers, le score au MMSE devait être supérieur ou égal à $28 / 30$ et ils ne devaient pas présenter de troubles cognitifs.

\subsection{Protocole expérimental}

Nous avons cherché à montrer la robustesse de l'influence des morphèmes lexicaux et dérivationnels lors d'une tâche d'inclusion catégorielle, auprès d'une population de sujets atteints de maladie d'Alzheimer. Nous avons délibérément abordé la catégorisation, non pas d'après le modèle taxonomique habituel (Rosch, 1978; Rosch \& Lloyd, 1978), mais à travers les informations véhiculées par la morphologie. Nous nous sommes appuyé sur des études montrant que les propriétés des signes linguistiques déterminent la structuration des catégories (Grinevald-Graig, op. cit., Dubois \& Poitou, op. cit.).

Notre hypothèse était que personnes atteintes de maladie d'Alzheimer utilisent essentiellement l'information fournie par la morphologie (lexicale et dérivationnelle) pour réaliser une tâche d'inclusion catégorielle (A. Devevey, Durand, Giroux, \& Midez, 2008; Giroux, 2007). L'objectif était de monter que, dès lors que l'on plaçait les sujets atteints de maladie d'Alzheimer non plus dans le contexte classique d'appartenance catégorielle sémantique d'après des caractéristiques perceptives (Montanes, Goldblum, \& Boller, 1996; Shallice, 1987; E.K. Warrington, 1975; E.K. Warrington \& Shallice, 1984; Weingartner, Kawas, Rawlings, \& Shapiro, 1993), mais dans un contexte de traitement d'indices à la fois purement linguistiques et implicites, leurs performances se rapprocheraient sensiblement de celles de sujets témoins appariés. En effet, toutes les études qui indiquent des différences nettes entre des échantillons malades Alzheimer vs témoins lors de tâche de jugement catégoriel classique, de type "est-ce qu'un x est un Y ?", ou encore "est-ce qu'un $x$ appartient à la catégorie des $Y$ ?" ; où $\mathrm{Y}$ et $\mathrm{x}$ entretiennent un rapport de type hyperonymie-hyponymie, appellent les sujets à faire référence à des connaissances lexico-sémantiques, donc sollicitent leurs capacités mnésiques sémantiques (explicites). Notre objectif était, au contraire, en adaptant les tâches classiquement proposées aux malades, de soumettre les sujets à une tâche qui les contraigne à traiter implicitement des indices morphologiques d'appartenance catégorielle, donc à solliciter leurs capacités mnésiques procédurales (implicites). Il s'agissait "d'expérimentaliser" le postulat selon lequel il existe en langue des marques des processus cognitifs de catégorisation (Grinevald-Graig, op. cit., Poitou \& Dubois, op. cit.).

A cette fin, nous avons utilisé des lexies ${ }^{3}$ constituées ou non du morphème "-ier". Celui-ci présente l'avantage, d'une part d'être constituant d'un grand nombre de lexèmes et de posséder une certaine variété sémantique d'autre part. Nous nous sommes basés sur deux de ces valeurs : celle d'arbre (fruitier) et de métier (agent).

Tous les sujets ont été confrontés à une tâche d'inclusion catégorielle orale dont la consigne de départ était: " Je vais vous dire des mots et vous me direz si c'est un nom d'Y ou pas? Il se peut qu'il y ait des mots que vous ne connaissiez pas; à ce moment là, on essaiera de voir s'il peut être oui ou non un nom d'Y. Par exemple, est-ce que le mot infirmier est un nom de métier? » Nous avons délibérément employé la formule "mot $x$, nom de $Y^{\prime \prime}$ (où x était le mot proposé et Y la catégorie : "arbre" ou "métier", selon la 
liste) afin de placer le sujet dans une tâche linguistique. Pour chaque mot, la consigne suivante était redonnée. Pour montrer les effets du suffixe et du radical au sein de la tâche d'inclusion catégorielle, nous avons déterminé 3 variables dépendantes ${ }^{4}$ :

\section{1. appartient ou non à la catégorie :}

(A) "arbre", (M) "métier", (NA) "non arbre", (NM) "non métier"

2. "avec suffixe-ier", "sans suffixe-ier" :

(AS) "avec suffixe", (SS) "sans suffixe"

3. radical lié ou pseudo-lié au fruit/à l'objet ${ }^{5}$ ou non lié :

(RL) "radical lié", (RNL) "radical non lié"

\begin{tabular}{|c|c|c|}
\hline & $\begin{array}{c}\text { Radical lié ou pseudo-lié } \\
\text { au fruit/à l'objet } \\
\text { (RL) }\end{array}$ & $\begin{array}{l}\text { Radical non lié } \\
\text { au fruit/à l'objet } \\
\qquad(\mathrm{RNL})\end{array}$ \\
\hline $\begin{array}{l}\text { Arbre ou Métier avec } \\
\text { suffixe "-ier" }(A A S-M \\
\text { AS })\end{array}$ & Ex $:$ cerisier / sabotier & Ex : peuplier / sommelier \\
\hline $\begin{array}{l}\text { Arbre ou Métier sans } \\
\text { suffixe "-ier" (A SS -M } \\
\text { SS) }\end{array}$ & Ex : airelle / pilote & Ex: sapin / architecte \\
\hline $\begin{array}{l}\text { Non-Arbre ou Non-Métier } \\
\text { avec suffixe "-ier" } \\
\text { (NAAS-NMAS) }\end{array}$ & Ex : anisier / vaisselier & $\begin{array}{l}\text { Ex : bélier, soulier } \\
\text { Sous-liste commune aux } \\
\text { protocoles "Arbres" et "Métiers" }\end{array}$ \\
\hline $\begin{array}{l}\text { Non-Arbre ou Non-Métier } \\
\text { sans suffixe -ier } \\
\text { (NA SS-NM SS) }\end{array}$ & Ex : maronite / lutin & Ex : licorne / cadastre \\
\hline
\end{tabular}

Tableau 1 : Plan expérimental de la constitution des huit listes de substantifs, exemple pour chaque liste.

Pour chaque catégorie, nous avons ainsi constitué au total 8 sous-listes croisant chacune ces 3 variables et constituées chacune de 10 substantifs (ou pseudo-mots pour la liste NA RL AS) ${ }^{6}$. La fréquence des lexies a été contrôlée (hormis, évidemment, pour la liste de pseudo-mots : NA RL AS). La variation de fréquence était comprise entre 1,581 et 2,691 $(\mathrm{m}=2,18)$, l'analyse à l'aide d'ANOVAs n'a pas montré de différence significative entre les sous-listes.

Les items "non arbre /non métier, radical non lié au fruit ou à l'objet avec suffixe -"ier " (NA ou NM RNL AS/ soulier) étaient communs aux 2 catégories, et constituaient ainsi une sous-liste retest entre la liste "Arbres " et la liste "Métiers". La fréquence des lexies choisies a été déterminée à l'aide de l'outil «Lexique 3», (New \& Pallier, 2001).

Ainsi, le protocole expérimental se déclinait sous la forme de 2 listes de 80 lexies chacune, équivalentes en fréquence. Nous nommerons désormais protocole "Arbres" la liste regroupant tous les items "arbre" et "non arbre", et protocole "Métiers", celle qui comporte tous les items "métier" et "non métier".

Pour les deux protocoles, les 80 items ont été répartis de manière pseudo-aléatoire tout en maîtrisant divers effets : variable radical, suffixe "-ier", fréquence, neutre... 
Pour chaque sujet, chacun des deux protocoles d'une durée de 10 à 20 minutes a été proposés à une semaine d'intervalle. Nous avons alterné l'ordre de passation, afin de contrôler d'éventuels effets d'entraînement ou d'amorçage.

L'ordre de passation des listes de chaque sujet contrôle était identique à celui du sujet cible apparié. Pour chaque item, la consigne «Est-ce que le mot $X$ est un nom d'Y? " était répétée autant de fois que nécessaire. Nous avons laissé à chacun des sujets le temps de répondre sans limitation de durée : s'il ne donnait pas de réponse, nous encouragions sa recherche en le sollicitant de nouveau. Une réponse était comptabilisée «juste » lorsque le sujet acceptait ou réfutait à bon escient l'appartenance du mot à la catégorie.

Seule la nature de la réponse juste ou erronée a été comptabilisée. Les tableaux ci-dessous présentent les moyennes du nombre de réponse exacte. Les différences entre les deux échantillons ont été mesurées à l'aide d'ANOVAs.

\subsection{Résultats}

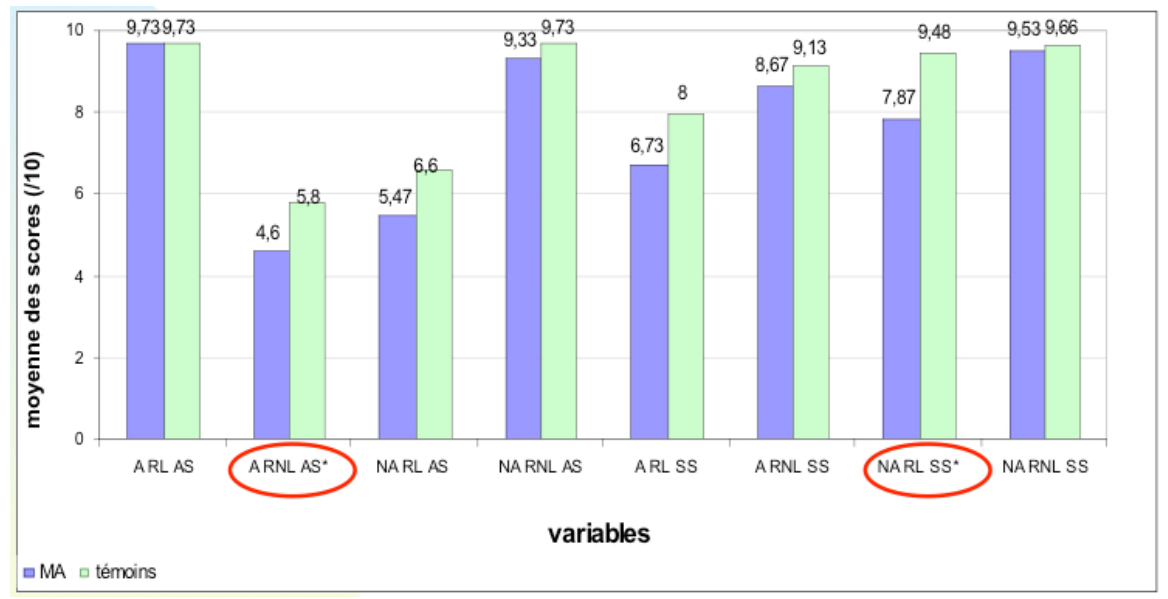

Figure 1 : Comparaison des scores aux sous-listes du protocole « Arbres » entre personnes atteintes de la maladie d'Alzheimer et témoins (*différence significative)

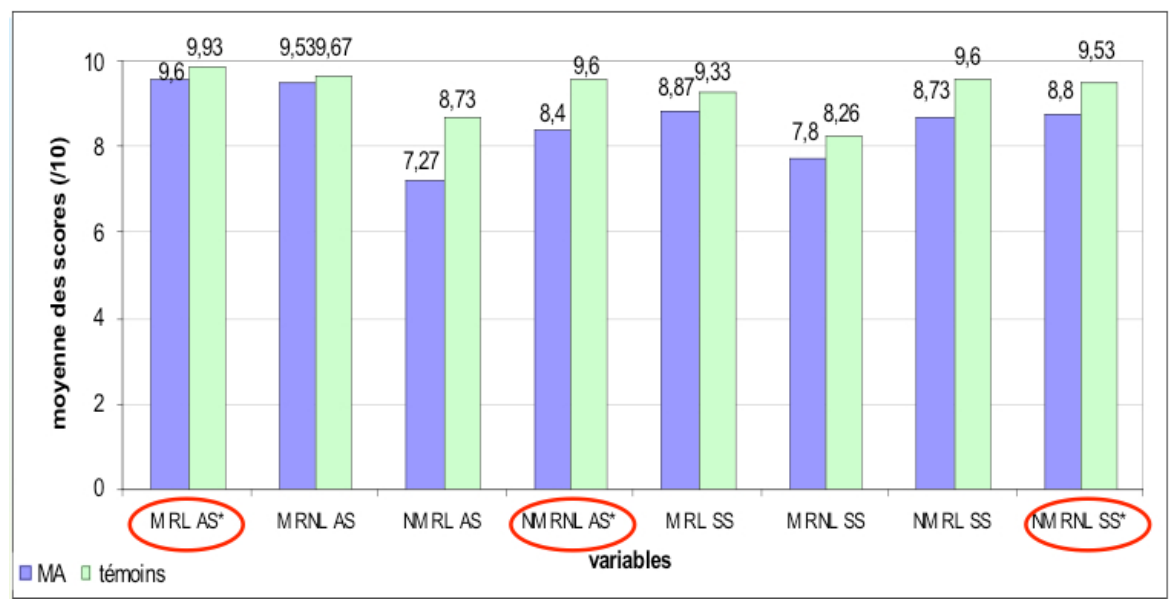

Figure 2 : Comparaison des scores aux sous-listes du protocole « Métiers » entre personnes atteintes de la maladie d'Alzheimer et témoins (*différence significative) 
L'analyse détaillée par sous-listes (figures 3 et 4) indique que les scores des personnes atteintes de la maladie d'Alzheimer ne se différencient significativement de ceux des témoins que pour 5 des 16 souslistes. Pour les arbres, il s'agit des listes arbres - radical non lié - avec suffixe "-ier" (A RNL AS, du type "peuplier" ; F $(1,28)=6,3 ; \mathrm{p}=.0181)$, non arbre - radical lié - sans suffixe "-ier" (NA RL SS, du type "maronite" ; F $(1,28)=5,298 ; \mathrm{p}=.0290)$. Pour les métiers, les différences entre personnes atteintes de la maladie d'Alzheimer et témoins concernent les listes métiers - radical lié - avec suffixe "-ier" (M RL AS, du type "sabotier" ; F $(1,28)=5,147 ; \mathrm{p}=.0312)$, non métier - radical non lié - avec suffixe "-ier" (NM RNL AS, du type "bélier" ; F $(1,28)=5,478 ; \mathrm{p}=.0266)$ et non métier - radical non lié - sans suffixe "-ier" (NM RNL SS, du type "licorne" ; F $(1,28)=4,321 ; \mathrm{p}=.0469)$. Toutefois en observant les résultats plus attentivement, on constate que la liste M RL AS, du type "sabotier", révèle un score moyen de 9,6/10 pour les personnes atteintes de la maladie d'Alzheimer, contre 9,93/10 pour les témoins. On peut considérer qu'une variation significative sur des scores aussi élevés n'est pas de nature à distinguer en pratique, les personnes atteintes de la maladie d'Alzheimer des témoins. Par ailleurs, la liste A RNL AS, du type "peuplier" indique un score proche du hasard pour les deux échantillons (4,6/10 pour les personnes atteintes de la maladie d's, contre 5,8/10 pour les témoins). Les scores à cette liste, tout comme ceux de la liste NA RL AS, du type "anisier", ne sont pas de nature à distinguer clairement les deux échantillons. Ainsi on peut considérer que les personnes atteintes de la maladie d'Alzheimer se distinguent réellement des témoins que pour trois listes sur seize uniquement.

Notre propos n'est pas ici de développer les caractéristiques de ces trois listes, propres à expliquer les processus cognitifs différents que chacune d'elles suscite chez les sujets, selon qu'elles les contraignent à recourir à un traitement lexico-sémantique (explicite) ou morphologique (implicite) ${ }^{7}$. Retenons simplement de cette étude que chaque fois que les items impliquent un processus implicite ou a contrario qu'ils suscitent un conflit entre traitement explicite et implicite (un cerisier est bien un arbre à cerises, en revanche un peuplier n'est pas un "arbre à peuple"), les deux échantillons ne se distinguent pas pour ce qui concerne le taux de réussite à la tâche proposée.

Il est néanmoins intéressant d'observer l'effet de la modalité sur certaines variables. La modalité (arbre/métier) influe essentiellement sur deux types de variables : RNL AS, du type "peuplier" et "sommelier" d'une part et RL AS, du type "anisier" et "vaisselier" d'autre part, essentiellement pour des raisons de contraintes antagonistes sollicitant les traitements implicite et explicite que nous ne développerons pas ici ("vaisselier" est attesté, alors que "anisier" ne l'est pas et "peuplier" n'a pas le même statut que "sommelier"). En revanche, il est remarquable de constater qu'une même liste (non métier/non arbre - radical non lié - avec suffixe "-ier"; NM/NA RNL AS, du type "bélier") ne suscite pas les mêmes comportements de la part des personnes atteintes de la maladie d'Alzheimer, selon qu'elle est traitée dans la modalité arbres ou dans la modalité métier. On peut ici trouver des arguments en faveur d'un effet de contrainte irrépressible de la consigne, qui ne permettrait pas de se libérer totalement d'un traitement explicite de la tâche proposée.

On peut néanmoins conclure que sur le plan des processus de traitement implicite du langage (versant réception), l'influence de la morphologie est indéniable. Cette influence conduit les deux échantillons à adopter des stratégies d'inclusion catégorielle similaires, alors qu'une tâche d'inclusion "ordinaire" les distinguerait de façon radicale.

\section{Expérience 2 : Importance de la morphologie dans la construction des néoformes chez des sujets porteurs de la maladie d'Alzheimer}

Ces résultats nous ont conduit à pousser davantage nos investigations. En effet, si les personnes atteintes de maladie d'Alzheimer conservent une capacité de décodage des marques morphologiques, dans quelle mesure sont-elles capables de les produire ? Lorsqu'au stade sévère de l'évolution, les troubles du langage ne se manifestent plus seulement par un manque du mot, on observe l'apparition de productions qui 
n'appartiennent apparemment pas à langue et que les neurologue ou les psychologues nomment paraphasies. Nous préférerons le terme de néoformes au sens de Dubois (J. Dubois et al., 1999) ${ }^{8}$. Le but de cette deuxième étude est de montrer que ces formes obéissent aux règles de construction morphologique de la langue. La signification des morphèmes grammaticaux reste un sujet de débat (Rastier, 2005 ), pourtant leur signification est largement documentée. Partant, si nous considérons à l'instar de Rastier que les morphèmes grammaticaux ont un sens, alors loin d'être vides de sens comme le décrit la littérature, les néoformes sont probablement le mode d'expression fonctionnel encore à la portée des malades au stade sévère, preuve d'un traitement préservé du "milieu langage" dans lequel ils évoluent. D'où l'intérêt de les recueillir et d'en observer le mode d'installation dans un corpus recueilli sur plusieurs mois.

\subsection{Protocole expérimental}

Notre étude s'est appuyée sur une analyse longitudinale de corpus. Une patiente atteinte de maladie d'Alzheimer au stade sévère, a été enregistrée lors d'échanges spontanés durant plusieurs mois. Le thème de l'échange était amorcé par la patiente. Les enregistrements d'une durée de 20 à 30 minutes ont été recueillis à l'aide d'un enregistreur discret pour être transcrits puis analysés. Toutes ces conditions étant rassemblées pour obtenir un corpus relevant d'un échange le plus naturel possible, au plus proche des capacités de la patiente.

Les corpus ainsi recueillis ont été analysés à l'aide du logiciel CLAN (Computerized Language Analysis), issu du projet de recherche international sur l'acquisition du langage CHILDES. Sept enregistrements ont été recueillis sur 15 mois, en avril 2006; septembre 2006 (b 0406); décembre 2006 (b 1206); mars 2007 (b 0"07) ; mai 2007 (b 0507); juin 2007 (b 0607); et juillet 2007 (b 0707). Lors des premiers enregistrements la patiente était âgée de 81,9 ans. Les effets de taille de corpus ont été neutralisés en considérant le nombre de mots (tokens) par énoncé.

\subsection{Résultats}

L'analyse met en évidence une diminution du nombre de mots par énoncé. Cette diminution concerne toutes les classes grammaticales, mais elle n'est significativement corrélée qu'avec le nombre de noms $($ rho $=.857 ; \mathrm{p}=.0358)$, de verbes $($ rho $=.929 ; \mathrm{p}=.0229)$, de pronoms (rho $=.857 \mathrm{p} .0358)$ et de prépositions par énoncé (rho $=.964 ; \mathrm{p}=.0182$ ). Elle n'est pas significative pour le nombre de déterminants et de conjonctions, d'adjectifs et d'adverbes par énoncé.

Les verbes et les pronoms sont les deux catégories grammaticales les plus représentées par énoncé. Les prépositions et les conjonctions (marqueurs de complexité syntaxique) apparaissent en très faible quantité dans les énoncés. 


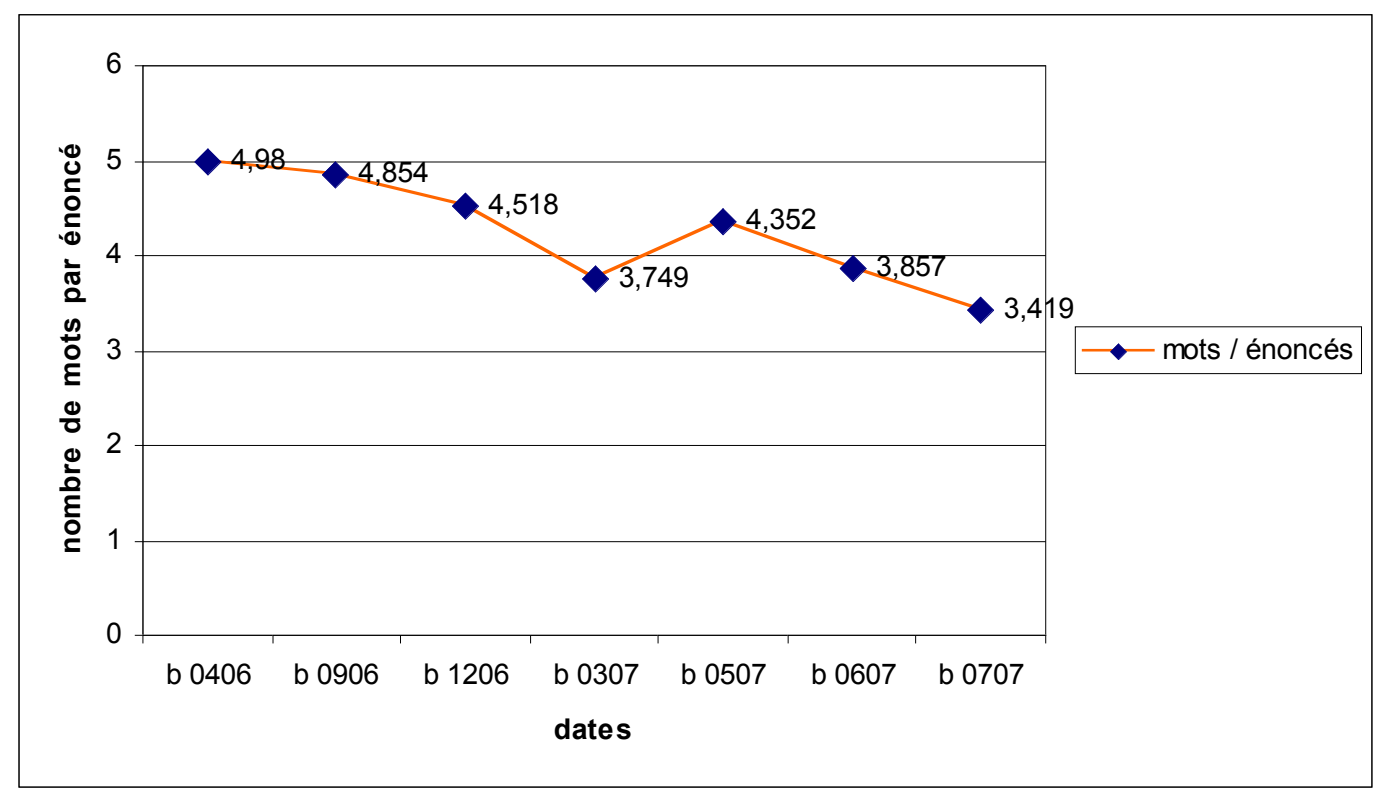

Figure 3 : Corpus de Mme B., évolution du nombre de mots (Token) par énoncés d'avril 2006 à juillet 2007

De cet aperçu, on retient que le vocabulaire s'appauvrit à mesure de l'évolution de la maladie (ce que confirment les données de la littérature). Cependant, dans le même temps, le nombre des néoformes augmente.

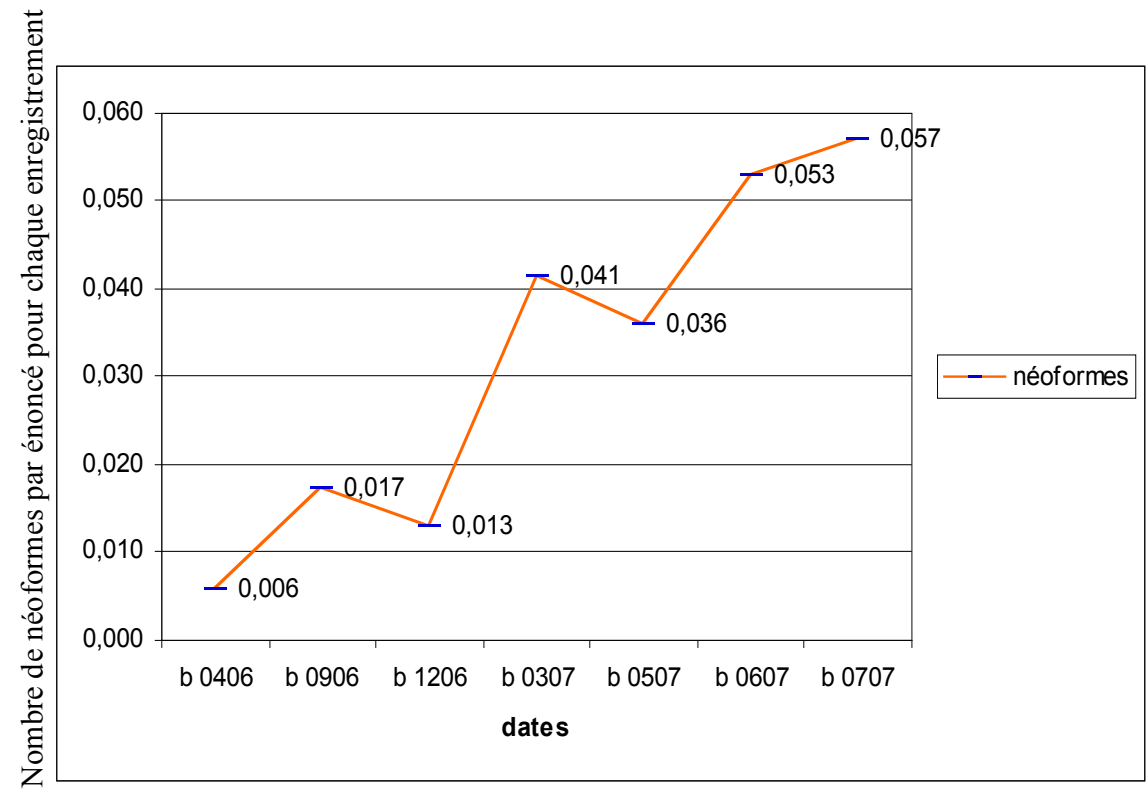

Figure 4 : Corpus de Mme B., évolution du nombre de néoformes (Token) par énoncés d'avril 2006 à juillet 2007 
Les conditions d'apparition de ces néoformes sont consécutives à l'augmentation du manque du mot. Elles apparaissent en compensation du manque d'une forme attestée, mais en conservent la fonction grammaticale. Les contextes d'apparition étant, en apparence, parfaitement aléatoires.

En fonction de sa place dans l'énoncé, de sa position dans le syntagme dans laquelle elle apparaît et de sa fonction sémantique présumée, chacune de ces néoformes a pu être répertoriée dans une classe grammaticale. Par exemple : «je veux pas caper» = verbe à l'infinitif; "c'est des gnoquets »= nom. Nous avons pris soin de vérifier que ces productions n'étaient attestées en français ni dans les différents dialectes de l'Isère ou de la région lyonnaise.

De ce classement, il appert que toutes les néoformes sont susceptibles d'être incluses dans une des classes "grammaticales" suivantes :

Noms ${ }^{9}$ : Exemple : le bardon, du vêquenet, un prédeau, une cabène, des gnoquets, un épineau / des épineaux...

Verbes du $1^{\mathrm{er}}$ groupe : réaforcher, dégularer, s'éberacule

Adjectifs qualificatifs $^{10}$ : cracheux...

Adverbes : gapin

Cependant, ces néoformes n'étant pas attestées par définition, nous préférons parler de pseudo classes grammaticales et utiliser les termes : néoforme nominale, néoforme verbale, néoforme adjectivale, néoforme adverbiale, plutôt qu'utiliser le nom usuel des classes grammaticales.

La répartition des néoformes produites pour chaque corpus, s'établit alors comme suit en pourcentages cumulés :

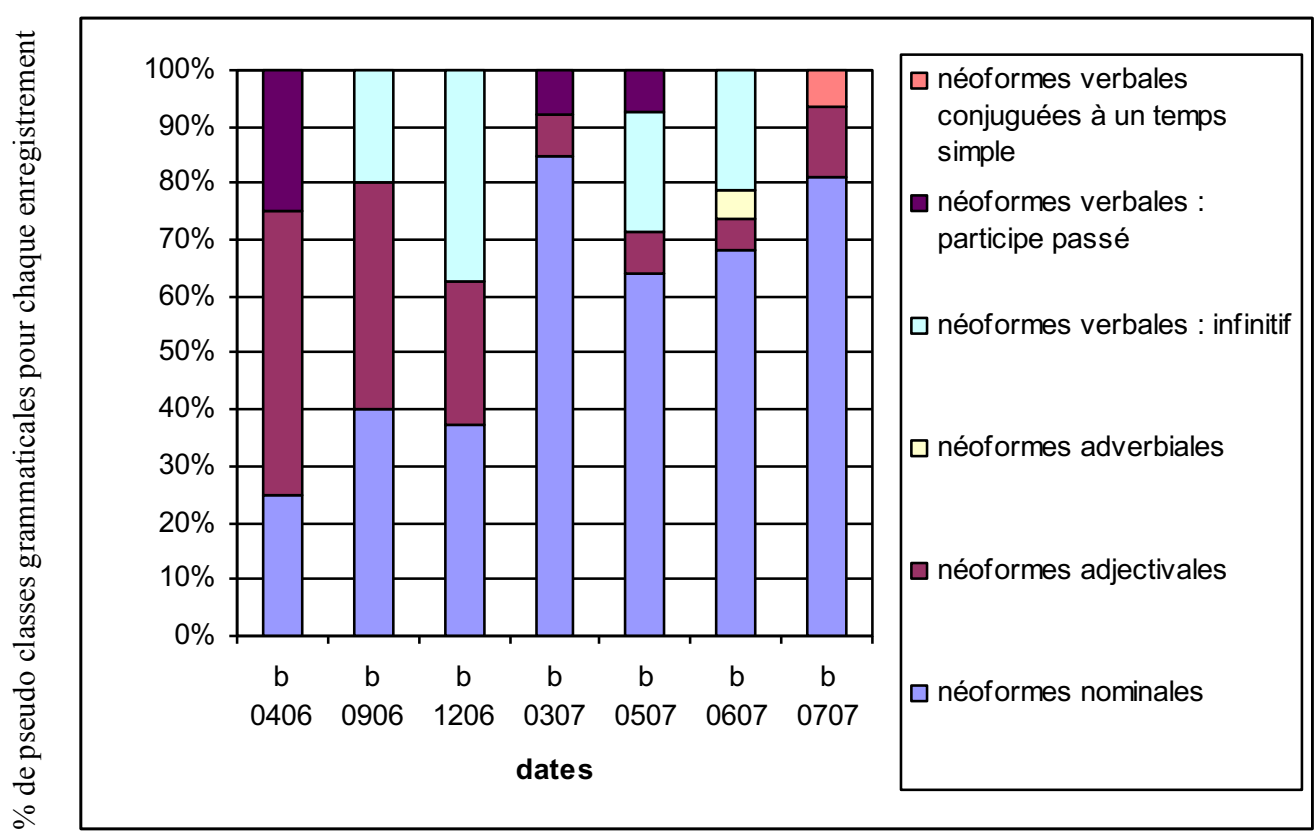

Figure 5 : Corpus de Mme B., évolution de la répartition des néoformes en \% cumulés en pseudo-classes grammaticales des néoformes d'avril 2006 à juillet 2007 
Cet histogramme nous permet de remarquer que chaque enregistrement comprend quatre pseudo-classes grammaticales différentes qui n'apparaissent pas toujours toutes de façon concomitante ni dans les mêmes proportions : la proportion de pseudo-noms augmente dans le même temps que la proportion de pseudoadjectifs diminue alors que le nombre de pseudo-verbes semble lui aussi diminuer.

Nous porterons une attention particulière aux néoformes nominales et verbales, qui constituent les pseudo-classes les plus représentées dans le corpus.

Les néoformes nominales sont essentiellement monomorphémiques. Les néoformes verbales nous apportent l'évidence du recours à des processus morphologiques flexionnels implicites. Il est possible de référer chaque néoforme à un groupe verbal : les néoformes verbales recensées dans le corpus sont formées sur le modèle des verbes du premier groupe. Ainsi, Mme B se réfère à la morphologie des verbes du premier groupe pour construire ses néoformes verbales sans doute parce que les verbes du premier groupe sont à la fois les plus nombreux, et les plus réguliers. On trouve des néoformes verbales à l'infinitif (les plus nombreuses), néoformes verbales conjuguées au "présent", néoformes verbales conjuguées au "participe passé".

On ne trouve dans le corpus qu'une seule néoforme verbale pouvant s'apparenter à un autre groupe : "plénir" ("vous m'avez pléni... ; il faut pas plénir").

Ainsi, les néoformes verbales apportent la preuve de la persistance d'une compétence linguistique implicite.

Les néoformes adjectivales apparaissent en nombre très restreint. Si on relève des marques flexionnelles ("crach + eux" par exemple), on ne relève aucune dérivation (de genre par exemple).

Pour conclure avec l'étude des néoformes, il apparaît qu'elles sont sémantiquement interprétables par le locuteur. Essentiellement en ce que, sur le plan discursif, elle participent indiscutablement à la progression narrative. Il est remarquable de constater, que contrairement à ce que l'on peut observer dans le discours de Mme B., recueilli à un stade modéré de sa maladie, où le récit était interrompu par des pauses importantes dues au manque du mot, les néoformes apparaissent au stade sévère dans le discours, sans être précédées d'aucune pause ni hésitation. Tout se passe comme si, à ce stade, le manque du mot était dépassé, compensé par le recours aux néoformes. Comme si les processus explicites étant désormais inhibés par l'avancée des lésions cérébrales, les processus implicites prenaient le relais pour compenser le déficit lexical. L'augmentation du nombre des néoformes et leur morphologie particulière qui les éloigne radicalement de simples suites syllabiques, en constituent le témoignage. Celles-ci sont à considérer non pas comme la marque d'une désintégration irréversible de la "fonction langage", mais comme de véritables mécanismes de compensation qui revêtent les caractéristiques morpholexicales et morphosyntaxiques de la langue.

\section{Discussion}

Si ces deux études tendent à révéler la robustesse des mécanismes cognitifs implicites impliqués dans le traitement de la morphologie, les enseignements qu'elles suggèrent sont de nature différente.

Dans le premier cas, la relative homogénéités des résultats obtenus chez les malades comme chez les contrôles remet en cause la pertinence des protocoles psycholinguistiques utilisés pour l'évaluation des troubles langagiers chez les personnes porteuses de maladie d'Alzheimer et, plus largement, de troubles cognitifs. Une évaluation du lexique basée sur les tâches lexicales et catégorielles classiques aurait sans aucun doute distingué les deux échantillon de manière plus radicale. Cependant en quoi cette expérience est-elle de nature à reconsidérer la vision modulaire du langage sur laquelle se fondent ces tâches classique ? Tout d'abord la morphologie est ordinairement évaluée au travers des tâches explicites de complètement de phrases ou de jugement de grammaticalité. La visée de l'expérience 1 est autre, en ce qu'elle tente de mobiliser les mêmes potentiels implicites que ceux mis en œuvre dans une situation 
d'interlocution au quotidien. Certes, en raison des contraintes d'analyse des résultats, la distinction entre procédure utilisée et une procédure modulaire classique peut sembler ténue. En réalité la différence entre les deux réside dans la démarche qui sous tend chacune d'elles. La procédure "classique" s'inspire de la démarche sémasiologique utilisée par les sciences cognitives : "Prenant un signifiant comme invariant, elle cherche à interdéfinir ses diverses acceptions, — comme jadis l'ont fait jadis Katz et Fodor (1963) [...]" (Rastier, 2005, p.138). C'est cette démarche qui impose une vision modulaire du langage. La démarche que nous avons adoptée est la démarche onomasiologique laquelle "part du signifié pour discerner les variétés synchroniques et les variations diachroniques du signifiant" (Rastier, op. cit. p. 138). Concernant la morphologie c'est cette opposition de démarche que traduisent les résultats de cette étude. Peu importe le statut de la lexie, c'est le processus interprétatif implicite de confrontation ou de complémentarité du radical et du suffixe qui est ici révélé. Ces mécanismes sont la preuve non pas d'une altération de la fonction langage (car alors il faudrait supposer une altération chez les témoins) mais d'une capacité cognitive préservée à traiter le "milieu langage".

C'est également cette démarche qui sous-tend l'expérience 2, sa visée est de révéler les productions habituellement qualifiées de déviantes des personnes atteintes de maladie d'Alzheimer, comme l'expression de signifiés, dont la valeur sémantique est révélée à la fois par le contexte et par la production des marques morphologiques. Elles sont, dans ce corpus, essentiellement flexionnelles. L'intérêt de la classification en catégorie grammaticale n'est absolument pas lexical, il réside dans la démonstration de la généralisation du phénomène à toutes les classes. Là encore, ces phénomènes langagiers non canoniques sont la manifestation de traitements cognitifs fonctionnels du "milieu langage"

Cette capacité est le reflet des capacités de compensation des malades. Elle n'a rien à voir avec l'innéité le la "fonction langage". Les difficultés en mémoire sémantique leur interdisant l'accès à la référence (explicite), le signifié va s'exprimer à travers une création, rendue possible par le recours à une procédure implicite, et dont la morphologie grammaticale va être la manifestation sémantique. Elle doit être considérée comme une variation singulière au même titre que les variations qui caractérisent le langage considéré comme milieu.

\section{Conclusion}

Ces deux études tendent à démontrer que les productions, apparemment dépourvues de sens, peuvent en réalité être considérées comme des formes idiolectales. Celles-ci restent dépendantes, même au stade ultime de l'évolution de la maladie, des règles de la langue. Elles sont la manifestation de ces règles, implicitement construites et intégrées, mémorisées par chaque sujet, en confrontation avec les variations du langage, considéré comme milieu au sein duquel il évolue.

Par ailleurs ces résultats tendent à affirmer l'hypothèse selon laquelle le paradigme qui s'appuie sur la théorie modulaire du langage combiné à une théorie d'une organisation catégorielle et taxonomique du lexique ne semble plus de nature à permettre la description ni la compréhension des processus de modification du langage au cours de l'évolution de la maladie d'Alzheimer. Ainsi, les difficultés lexicosémantiques des personnes atteintes de la maladie d'Alzheimer ne peuvent être pensées uniquement en termes de difficulté d'accès ou de perte des représentations sémantiques ainsi que le suppose la psychologie cognitive. Elles ne peuvent davantage être considérés comme une "perte du langage" voire un "désapprentissage de la langue", mais comme un type singulier de variation, reflet du processus de compensation à la progression des lésions neuronales. 


\section{Références bibliographiques}

Baayen, R. H. (2007). Storage and computation in the mental lexicon. In G. J. a. G. Libben (Ed.), The Mental Lexicon: Core Perspectives (pp. 81-104): Elsevier.

Barkat-Defradas, M., \& Gayraud, F. ( 2013). Maladie d'Alzheimer et bilinguisme. Rééducation Orthophonique,, $253,31-53$

Barkat-Defradas, M., Martin S., Rico-Duarte, L., \& Brouillet, D. (2008). Les troubles dulangage dans la maladie d'Alzheimer :. Paper presented at the 27 journ ee d' etudes sur la Parole, Avignon, France. .

Berrewaerts, J., Hupet, M., \& Feyereisen, P. (2002). Analyse psycholinguistique de l'expression orale dans les stades précoces de la maladie d'Alzheimer: comparaison de quatre tâches. Le Langage et l'Homme, 37( 2), 119-138.

Chomsky, N. (1955). The logical structure of linguistic thory. Masschusetts Institute of Technology New York and London: Plenum Press, 1975; Chicago: University of Chicago Press, 1985.

Chomsky, N. (2011). Language and Other Cognitive Systems. What Is Special About Language ? Language Learning and Development, 7(4), 263-278.

Devevey, A. (2008). Le langage dans les maladies du vieillissement Maladies du vieillissement... un exercice d'interdisciplinarité (pp. 55-75). Lausanne: Université de Lausanne.

Devevey, A. (2009a). Préservation des aspects implicites (morphologiques) du langage dans les maladies neurodégénératives Paper presented at the Entretiens d'Orthophonie 2009 Paris.

Devevey, A., Durand, E., Giroux, J., \& Midez, P. (2008). Que nous révèle la préservation des capacités morphosyntaxique des capacités sémantiques ? In Publibook. (Ed.), Xème colloque international sur le vieillissement cognitif. Paris, : Actualités Collection Psychologie cognitive, EPU.

Dubois, D. (Ed.) (1991). Sémantique et cognition: catégories, prototypes, typicalité: Éditions du Centre national de la recherche scientifique.

Dubois, D., \& Poitou, J. (2002). Des « normes catégorielles »: structuration cognitive et/ou linguistique des catégories sémantiques. Intellectica, 2002/,, 35,(2), 217-249.

Dubois, J., Giacomo, M., Guespin, L., Marcellesi, J. B., \& Mével, J. P. (1999). Dictionnaire de Linguistique et des Sciences du Langage. Paris: Larousse-Bordas/HER.

Fodor, J. A. (1983). The modularity of mind : an essay on faculty psychology. Cambridge, Mass.: MIT Press.

Folstein, M. F., Folstein, S. E., \& P.R., M. H. (1975). "Mini Mental State" : a practical method for grading the cognitive state of patients for the clinician. Journal of Psychiatric Research, 12, 189-198.

Giroux, J. (2007). Recherche d'un processus d'inclusion catégorielle par les morphèmes lexicaux et dérivationnels chez des patients atteints de maladie d'Alzheimer. (Mémoire pour obtenir le Certificat de Capacité d'Orthophoniste), Université de Franche-Comté, Besançon.

Grinevald-Graig, C. (1986). Jacaltec Noun Classifiers.A Study in Gramaticalization. Lingua, 70, 241-284.

Kavé, G., \& Lévy, Y. (2004). Preserved Morphological Decomposition in Persons With Alzheimer's Disease. Journal of Speech, Language, and Hearing Research, 47 (August 2004), 835-847.

Kempler, D., Curtiss, S., \& C., J. (1987). Syntactic preservation in Alzheimer's disease Journal of Speech and Hearing Research, 30, 343-350.

Macoir, J., \& Fossard, M. (2008). Mémoire procédurale et processus cognitifs : application de règles en morphologie, syntaxe et calcul arithmétique Paper presented at the Congrès Mondial de Linguistique Française - CMLF'08.

Montanes, P., Goldblum, M.-C., \& Boller, F. (1996). Classification deficits in Alzheimer's disease with special reference to living and nonliving things. Brain and Language, 54(2), (335-358).

Murdoch, B. E., \& Chenery, H. J. (1987). Language Disorders in Dementia of theAlzheimer Type. Brain and Language, 31, 122-137. 
Nespoulous, J.-L. (1994). Linguistique, neurolinguistique et neuropsycholinguistique. Un parcours en quatre étapes.... In X. Séron \& M. Jeannerod (Eds.), Neuropsychologie Humaine (pp. 317-319). Bruxelles: Mardaga.

Nespoulous, J.-L. (2004). Linguistique, Pathologie du Langage et Cognition : des dysfonctionnements langagiers à la caractérisation de l'architecture fonctionnelle du langage. In J. F. Catherine Fuchs, Jean-François Le Ny,Jean-Luc Nespoulous,Collectif (Ed.), La linguistique cognitive (pp. 171-194): Ophrys \& Editions de la MSH.

New, B., \& Pallier, C. (2001). LExique 3. from RISC CNRS http://www.lexique.org/

Paradis, M. (1994). Neurolinguistic Aspects of Implicit and Explicit Memory: Implications for Bilingualism and SLA. In N. E. (ed.) (Ed.), Implicit and explicit learning of languages (pp. 393-419). London: Academic Press.

Paradis, M. (2004). A neurolinguistic theory of bilingualism. Amsterdam: John Benjamins Publishing Compagny.

Pinker, S. (1999). Words and rules: The ingredients of language. London: Weidenfeld \& Nicolson. .

Pinker, S., \& Ullman, M. T. (2002). The past and future ofthe past tense. TRENDS in Cognitive Sciences, 6(11), 8.

Poitou, J., \& Dubois, D. (1999). Catégories sémantiques et cognitives. Cahiers de lexicologie, 74, 15-27.

Rastier, F. (1987). Sémantique interprétative. Paris: Presses Universitaires de France.

Rastier, F. (2003). Le langage comme milieu : des pratiques aux oeuvres. Texto!Textes \& Cultures. Retrieved from http://www.revue-texto.net/index.php?id=545.

Rastier, F. (2005). De la sémantique cognitive à la sémantique diachronique : les valeurs et évolutions des classes lexicales. . Texto ! Retrieved from http://www.revue-texto.net/Inedits/Rastier/Rastier_Classes$\underline{\text { lexicales.html }}$

Rastier, F. (2005). La microsémantique. texto !

, vol. $X, 1-46$. Retrieved from http://www.revue-texto.net/Inedits/Rastier/Rastier_Microsemantique.html

Rosch, E. (1978). Principle of categorisation. In E. R. B. Lloyd (Ed.), Cognition and categorisation (pp. 27-48). Hillsdale (N.-J.): L. Erlbaum.

Rosch, E., \& Lloyd, B. (1978). Cognition and categorization. Hillsdale (N.-J.): L. Erlbaum.

Rosch, E., Mervis, C. B., Gray, W. D., Johnson, D. M., \& Boyes-Braem, P. (1976). Basic Objects in Natural Categories. Cognitive Psychology, 8, 382-439.

Rossi, J. P. (2013). Psycho-neurologie du langage. Le sens des mots et des objets du monde. Bruxelles: De Boeck Solal.

Shallice, T. (1987). Impairments of semantic processing : multiple dissociations. In S. G. Coltheart M., Job R. (Eds.) (Ed.), The Cognitive Neuropsychology of Language. London: Lawrence Erlbaum Associates.

Warrington, E. K. (1975). The selective impairment of semantic memory. Quarterly Journal of Experimental Psychology., 27, 635-657.

Warrington, E. K., \& Shallice, T. (1984). Category specific semantic impairment. Brain, 107, 829-853.

Weingartner, H., Kawas, C., Rawlings, R., \& Shapiro, M. (1993). Changes in semantic memory in early stage Alzheimer's disease patients. GERONTOLOGIST CO: GRNTA, 33(5), (637-643). 
Annexe $1:$ Items du protocole expérimental

\begin{tabular}{|c|c|c|}
\hline \multicolumn{3}{|c|}{ Protocole "Arbres" } \\
\hline ARBRES & $\begin{array}{l}\mathrm{RL} \\
\text { Radical Lié }\end{array}$ & $\begin{array}{l}\text { RNL } \\
\text { Radical Non Lié }\end{array}$ \\
\hline \multirow{5}{*}{ A AS } & cerisier & peuplier \\
\hline & pommier & laurier \\
\hline & poirier & coudrier \\
\hline & manguier & palmier \\
\hline & prunier & gommier \\
\hline \multirow{5}{*}{$\begin{array}{l}\text { Arbre } \\
\text { Avec Suffixe }\end{array}$} & abricotier & tulipier \\
\hline & amandier & virgilier \\
\hline & noisetier & arganier \\
\hline & châtaignier & savonnier \\
\hline & figuier & micocoulier \\
\hline \multirow[t]{4}{*}{ moyennes } & $9,73 / 9,73$ & $4,6 / 5,8$ \\
\hline & airelle & sapin \\
\hline & sureau & frêne \\
\hline & kiwi & catalpa \\
\hline \multirow[t]{2}{*}{ A SS } & tilleul & orme \\
\hline & aubépine & saule \\
\hline Arbre & myrte & aulne \\
\hline \multirow[t]{4}{*}{ Sans $\underline{\text { Suffixe }}$} & eucalyptus & érable \\
\hline & mimosa & cèdre \\
\hline & lilas & épicéa \\
\hline & houx & platane \\
\hline \multirow[t]{4}{*}{ moyennes } & $6,73 / 8$ & $8,67 / 9,13$ \\
\hline & anisier & soulier \\
\hline & glutier & bélier \\
\hline & glandier & bouclier \\
\hline \multirow[t]{2}{*}{ NA AS } & aubépinier & clapier \\
\hline & grenadinier & clavier \\
\hline Non Arbre & ambrier & flibustier \\
\hline \multirow[t]{4}{*}{ Avec Suffixe } & goudronnier & gésier \\
\hline & coriandrier & fumier \\
\hline & safranier & épervier \\
\hline & mentholier & pluvier \\
\hline moyennes & $5,47 / 6,6$ & $9,33 / 9,73$ \\
\hline \multirow[t]{2}{*}{ NA SS } & maronite & licorne \\
\hline & fraiseur & gîte \\
\hline
\end{tabular}

\begin{tabular}{|c|c|c|}
\hline \multicolumn{3}{|c|}{ Protocole "Métiers" } \\
\hline METIERS & $\begin{array}{l}\mathrm{RL} \\
\text { Radical Lié }\end{array}$ & $\begin{array}{l}\text { RNL } \\
\text { Radical Non Lié }\end{array}$ \\
\hline \multirow{5}{*}{ M AS } & sabotier & sommelier \\
\hline & bottier & huissier \\
\hline & grainetier & croupier \\
\hline & bijoutier & meunier \\
\hline & forestier & ouvrier \\
\hline \multirow{5}{*}{$\begin{array}{l}\text { Métiers } \\
\text { Avec Suffixe }\end{array}$} & banquier & courtier \\
\hline & drapier & greffier \\
\hline & cafetier & palefrenier \\
\hline & serrurier & chancelier \\
\hline & postier & tenancier \\
\hline \multirow[t]{4}{*}{ moyennes } & $9,6 / 9,93$ & $9,55 / 9,67$ \\
\hline & pilote & architecte \\
\hline & arbitre & député \\
\hline & dramaturge & sage-femme \\
\hline \multirow[t]{2}{*}{ M SS } & pâtre & militaire \\
\hline & orfèvre & vigile \\
\hline Métier & garde-chasse & bosco \\
\hline \multirow[t]{4}{*}{ Sans $\underline{\text { Suffixe }}$} & vigneron & scribe \\
\hline & cinéaste & fantassin \\
\hline & nourrice & laquais \\
\hline & astronaute & canut \\
\hline \multirow[t]{4}{*}{ moyennes } & $8,87 / 9,33$ & $7,8 / 8,27$ \\
\hline & vaisselier & soulier \\
\hline & fourmilier & bélier \\
\hline & balancier & bouclier \\
\hline \multirow[t]{2}{*}{ NM AS } & chantier & clapier \\
\hline & pierrier & clavier \\
\hline \multirow{5}{*}{$\begin{array}{l}\text { Non Métier } \\
\text { Avec Suffixe }\end{array}$} & dentier & flibustier \\
\hline & poudrier & gésier \\
\hline & couscoussier & fumier \\
\hline & herbier & épervier \\
\hline & colombier & pluvier \\
\hline moyennes & $7,26 / 8,73$ & $8,4 / 9,6$ \\
\hline \multirow[t]{2}{*}{ NM SS } & lutin & cadastre \\
\hline & marinade & esthète \\
\hline
\end{tabular}




\begin{tabular}{|c|c|c|}
\hline Non Arbre & grenadine & limonade \\
\hline \multirow[t]{7}{*}{$\underline{\text { Sans }} \underline{\text { Suffixe }}$} & pommeau & tricycle \\
\hline & noyau & jonquille \\
\hline & mangouste & bêche \\
\hline & mandarin & fennec \\
\hline & glandeur & condor \\
\hline & murène & pivoine \\
\hline & poireau & arnaque \\
\hline moyennes & $7,87 / 9,46$ & $9,53 / 9,66$ \\
\hline
\end{tabular}

\begin{tabular}{|l|l|l|}
\cline { 2 - 3 } Non Mans $\underline{\text { Suffixer }}$ & barbote & misanthrope \\
\cline { 2 - 3 } & drogué & clochard \\
\cline { 2 - 3 } & polisson & binocle \\
\cline { 2 - 3 } & verrou & carrosse \\
\cline { 2 - 3 } & lampée & chapon \\
\cline { 2 - 3 } & chantre & aïeul \\
\cline { 2 - 3 } & textile & guilledou \\
\cline { 2 - 3 } & vaseline & carabin \\
\hline moyennes & $8,73 / 9,6$ & 8,63 \\
\hline
\end{tabular}

en bleu : moyenne des scores des patients / en vert : moyenne des scores des témoin

\footnotetext{
${ }^{1}$ Mémoire de travail, mémoire sémantique, fonctions exécutives principalement.

${ }^{2}$ Au sens de structuré comme tel dans le monde.

${ }^{3}$ Nous préférons le terme lexie en tant qu'unité fonctionnelle significative du discours, à lexème, en tant qu'unité unité abstraite appartenant à la langue (J. Dubois, Giacomo, Guespin, Marcellesi, \& Mével, 1999), afin de rendre compte des unités la catégorie "NA AS - NM AS" (cf. p. suivante) lesquelles, n'appartenant pas à la langue, ne peuvent être considérés comme des lexèmes.

${ }^{4}$ Avant chacun des items cités, se trouve entre parenthèses son abréviation en sigles, suivi dans le tableaul d'un exemple typique de la sous-liste concernée, $1^{\text {er }}$ exemple de chaque sous-liste du tableau en Annexe 1.

${ }^{5}$ Le terme "objet" réfère ici à l'objet principalement impliqué dans le travail

${ }^{6}$ Cette liste est constituée de lexèmes qui, dans le contexte de l'expérimentation, pourraient induire une interprétation du type "lié au fruit" ou "lié à l'objet" : "maronite" peut évoquer le "marron" du "marronnier" comme "marinade" peut évoquer "marin". Cf. Annexe 1 ci-dessous.

${ }^{7}$ Voir à ce sujet (A. Devevey et al., 2008; Giroux, 2007)

8 "termes qui ne correspondent à aucun mot de la langue et qui apparaissent à la place de mots normalement attendus dans le discours... ce sont des formes qui n'existent pas dans la langue mais dont la constitution phonique obéit aux règles morphophonologiques de la langue du locuteur. » (op. cit. p. 342).
}

${ }^{9}$ L'orthographe des néoformes présentées ici dépend, bien entendu, du choix des auteurs. Nous avons décidé pour des raisons d'analyse morphologique, de ne pas utiliser l'alphabet phonétique international, mais de transcrire chaque syllabe des néoformes à partir de l'orthographe la plus plausible et la plus courante dans un contexte donné.

${ }^{10}$ Désormais "adjectifs" dans cet article. 